archives

of thermodynamics

Vol. 37(2016), No. 3, 63-78

DOI: $10.1515 /$ aoter-2016-0020

\title{
Analytical 1D models of the wall thermal resistance of rectangular minichannels applied in heat exchangers
}

\author{
WITOLD RYBIŃSKI* \\ JAROSEAW MIKIELEWICZ
}

Institute of Fluid Flow Machinery, Polish Academy of Sciences, Fiszera 14, 80-231 Gdańsk, Poland

\begin{abstract}
The paper presents four 1-dimensional models of thermal resistance of walls in a heat exchanger with rectangular minichannels. The first model is the simplest one, with a single wall separating two fluids. The second model of the so called equivalent wall takes into account total volume of intermediate walls between layers of minichannels and of side walls of minichannels. The next two more complicated models take separately into account thermal resistance of these walls. In these two models side walls are treated as fins. The results of models comparison are presented. It is shown that thermal resistance may be neglected for metal walls but it should be taken into account for the walls made of plastics. For the case of non-neglected wall thermal resistance the optimum wall thickness was derived. Minichannel heat exchangers made of plastic are larger than those built of metal, but are significantly cheaper. It makes possible to use of such exchangers in inexpensive microscale ORC installations.
\end{abstract}

Keywords: Minichannel heat exchanger; Wall thermal resistance; Rectangular minichannel

\section{Nomenclature}

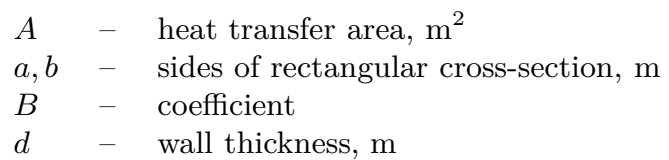

${ }^{*}$ Corresponding Author. E-mail: witold.rybinski@imp.gda.pl 


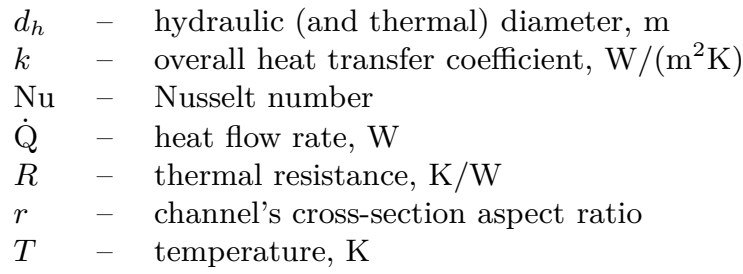

\section{Greek symbols}

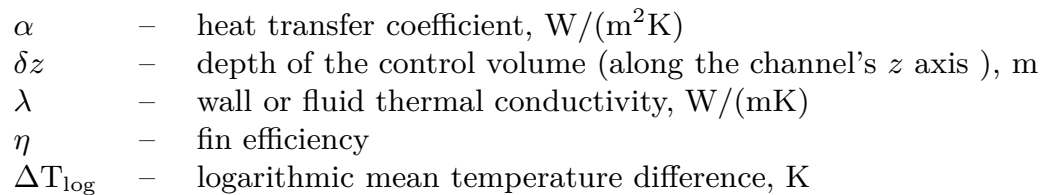

\section{Subscripts}

$$
\begin{array}{lll}
c, h & - & \text { cold or hot fluid } \\
e & - & \text { equivalent wall } \\
i, s, i+s & - & \text { intermediate, side or total intermediate wall } \\
w, f l & - & \text { wall or fluid conductivity }
\end{array}
$$

\section{Introduction}

Small scale organic Rankine cycle (ORC) installations demand use of miniature and inexpensive heat exchangers. Minichannel heat exchangers may be made of metal, but there is a possibility to use polymers or other formable materials of small thermal conductivity [1]. At the Institute of Fluid Flow Machinery PAS the design and investigation of miniature heat exchangers for application in ORC is carried out. It is planned to test some non-metal materials for operation in ORC installation. In the paper there are presented four 1-dimensional models of the wall thermal resistance in heat exchangers with rectangular minichannels of the same size. Two of these models are presented in [2]. The models were analysed and compared. It is shown that the thermal resistance of metal walls may be neglected. For the walls made of plastics these models show that the optimal wall thickness is relatively small. The models of the wall thermal resistance are used to calculate the overall heat transfer coefficient $k$ in heat exchangers with rectangular minichannels.

General view of a minichannel heat exchanger and considered control volume is presented in Fig. 1.

Heat transfer rate in the control volume is given by the formula:

$$
\dot{Q}=\frac{1}{R} \Delta T_{\log } \text {. }
$$



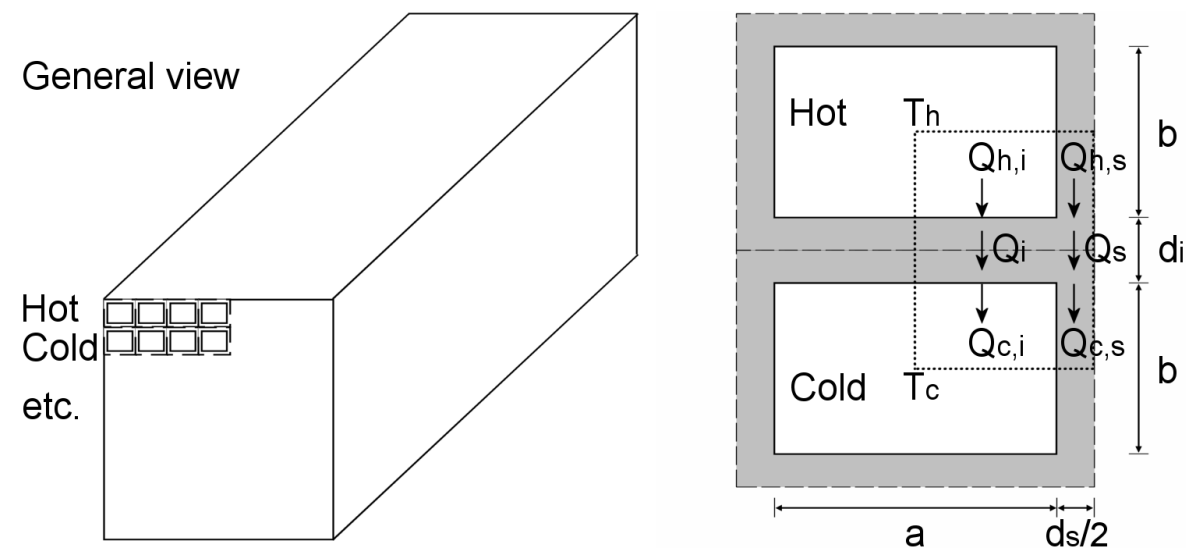

Figure 1: Schematic view of a heat exchanger's cross-section with layers of hot and cold minichannels and of the control volume (dotted line) with heat flows.

Total thermal resistance is given by the overall heat transfer coefficient and heat transfer area of the control volume (in the figure the control volume depth $\delta z$ is along the channel's $z$-axis, perpendicular to the figure's plane):

$$
R=\frac{1}{k A},
$$

where

$$
A=\frac{a+b}{2} \delta z .
$$

Partial thermal resistance are calculated in the same way, for example:

$$
R_{h, i}=\frac{1}{\alpha_{h} A_{h, i}},
$$

where

$$
A_{h, i}=\frac{a}{2} \delta z .
$$

Channel's cross-section aspect ratio is defined as

$$
r=\frac{a}{b} .
$$

Hydraulic and thermal diameter is given by

$$
d_{h}=\frac{2 a b}{a+b}=\frac{2 r}{r+1} b .
$$




\section{Model 1 - no side walls of the channels}

First the simplest model is considered where side walls of the channels are removed, Fig. 2.
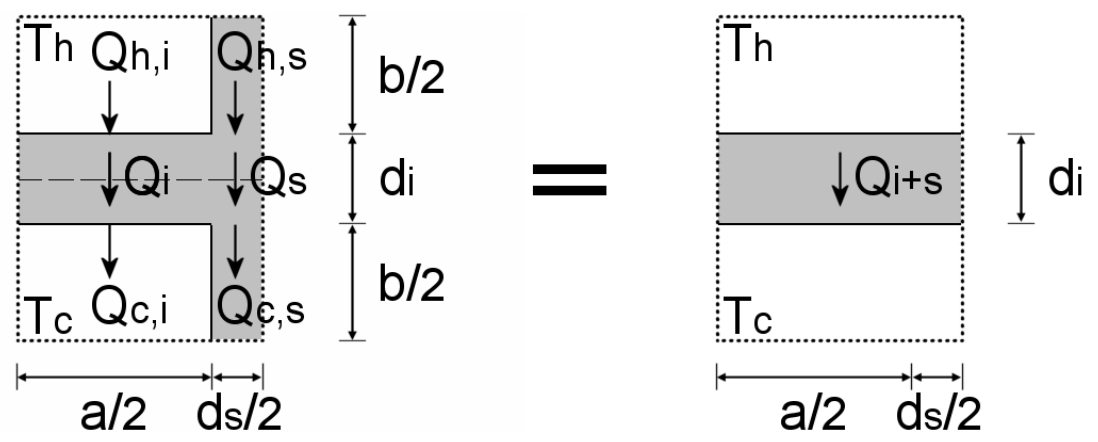

Figure 2: Model 1 - the side walls of the channels are removed.

The formula for total thermal resistance in the models 1 has the form

$$
R=R_{h}+R_{i+s}+R_{c}
$$

Use of (2) and (4) leads to the equation

$$
\frac{1}{k_{1} \frac{a+b}{2} \delta z}=\frac{1}{\alpha_{h} \frac{a+d_{s}}{2} \delta z}+\frac{1}{\frac{\lambda_{w}}{d_{i}} \frac{a+d_{s}}{2} \delta z}+\frac{1}{\alpha_{c} \frac{a+d_{s}}{2} \delta z},
$$

which gives the final formula for overall heat transfer coefficient $k_{1}$ :

$$
\frac{1}{k_{1}}=\frac{r+1}{r+d_{s} / b}\left(\frac{1}{\alpha_{c}}+\frac{1}{\lambda_{w} / d_{i}}+\frac{1}{\alpha_{c}}\right) .
$$

\section{Model 2 - walls are replaced by an equivalent wall of the same volume and area}

In model 2 the walls are represented by an equivalent wall of the same volume and area, Fig. 3. The formula (8), by use of (2) and (4), has the following form in this model

$$
\frac{1}{k_{2} \frac{a+b}{2} \delta z}=\frac{1}{\alpha_{h} \frac{a+b}{2} \delta z}+\frac{1}{\frac{\lambda_{w}}{d_{e}} \frac{a+b}{2} \delta z}+\frac{1}{\alpha_{c} \frac{a+b}{2} \delta z},
$$



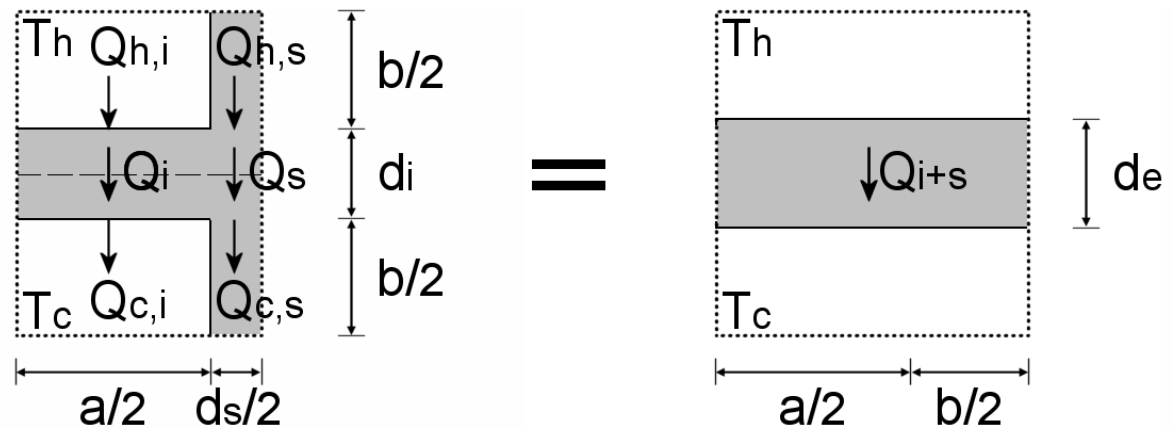

Figure 3: Model 2 - the walls are replaced by an equivalent wall of the same volume and area.

with the equivalent wall thickness

$$
d_{e}=\left(\frac{a+d_{s}}{2} d_{i}+2 \frac{d_{s}}{2} \frac{b}{2}\right) / \frac{a+b}{2}=\frac{\left(r+d_{s} / b\right) d_{i}+d_{s}}{r+1} .
$$

Simplification of (11) gives the final formula for $k_{2}$ :

$$
\frac{1}{k_{2}}=\frac{1}{\alpha_{c}}+\frac{1}{\lambda_{w} / d_{e}}+\frac{1}{\alpha_{c}} .
$$

\section{Model 3 - side walls as fins, one common resis- tance of the wall between hot and cold channels}

In this model heat transfer through the intermediate and side walls of the channels are taken separately into account whereas side walls are treated as fins. However, heat flows through the wall between hot and cold channels are not separated, Fig. 4. The formula for the total thermal resistance in the model 3 has the form

$$
R=\left(1 / R_{h, i}+1 / R_{h, s}\right)^{-1}+R_{i+s}+\left(1 / R_{c, i}+1 / R_{c, s}\right)^{-1} .
$$

Use of (2) and (4) leads to the equation

$$
\frac{1}{k_{3} \frac{a+b}{2} \delta z}=\frac{1}{\alpha_{h} \frac{a+\eta_{h} b}{2} \delta z}+\frac{1}{\frac{\lambda_{w}}{d_{i}} \frac{a+d_{s}}{2} \delta z}+\frac{1}{\alpha_{c} \frac{a+\eta_{c} b}{2} \delta z},
$$

with fin efficiency given by ( $B$ is introduced later)

$$
\eta=\frac{\tanh \left(\sqrt{\frac{\alpha b}{2 \lambda_{w}} \frac{b}{d_{s}}}\right)}{\sqrt{\frac{\alpha b}{2 \lambda_{w}} \frac{b}{d_{s}}}}=\frac{\tanh \left(\sqrt{B \frac{b}{d_{s}}}\right)}{\sqrt{B \frac{b}{d_{s}}}},
$$




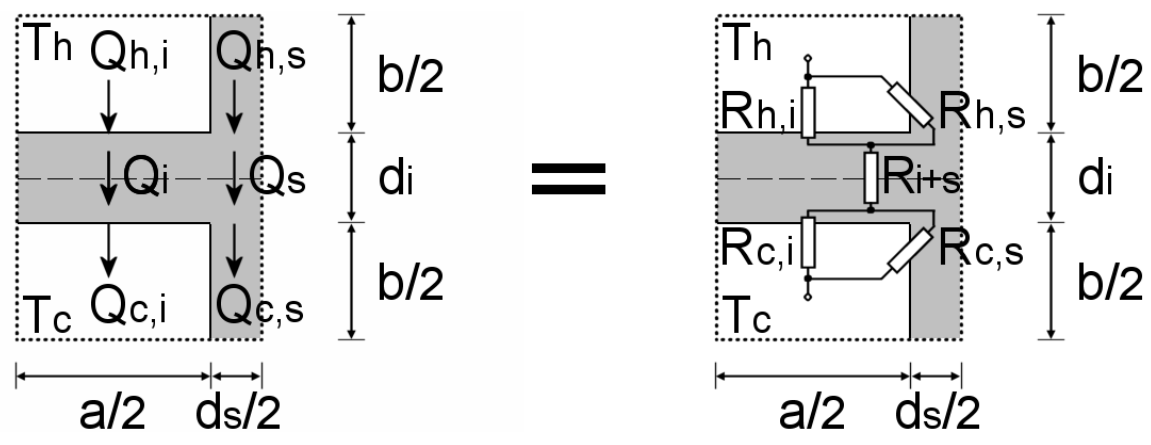

Figure 4: Model 3 - separated thermal resistances: channel side walls are treated as fins, one single common resistance of the wall between hot and cold channels.

Simplification of (15) gives the final formula for $k_{3}$ :

$$
\frac{1}{k_{3}}=\frac{1}{\alpha_{h} \frac{r+\eta_{h}}{r+1}}+\frac{1}{\frac{\lambda_{w}}{d_{i}} \frac{r+d_{s} / b}{r+1}}+\frac{1}{\alpha_{c} \frac{r+\eta_{c}}{r+1}} .
$$

\section{Model 4 - side walls as fins, two separate resistances of the wall between hot and cold channels}

In this model heat flows through the intermediate and side wall of the channels are taken separately into account whereas side walls are treated as fins. Heat flows through the wall between hot and cold channels are also separated into two parts, Fig. 5. The formula for the total thermal resistance in the model 4 has the form

$$
R^{-1}=\left(R_{h, i}+R_{i}+R_{c, i}\right)^{-1}+\left(R_{h, s}+R_{s}+R_{c, s}\right)^{-1} .
$$

Use of (2) and (4) leads to the equation:

$$
\begin{gathered}
k_{4} \frac{a+b}{2} \delta z=\left(\frac{1}{\alpha_{h} a / 2 \cdot \delta z}+\frac{1}{\lambda_{w} / d_{i} \cdot a / 2 \cdot \delta z}+\frac{1}{\alpha_{c} a / 2 \cdot \delta z}\right)^{-1}+ \\
+\left(\frac{1}{\alpha_{h} \eta_{h} b / 2 \cdot \delta z}+\frac{1}{\lambda_{w} / d_{i} \cdot d_{s} / 2 \cdot \delta z}+\frac{1}{\alpha_{c} \eta_{c} b / 2 \cdot \delta z}\right)^{-1}
\end{gathered}
$$

Simplification of (19) gives the final formula for $k_{4}$ :

$$
k_{4}=\frac{r}{r+1}\left(\frac{1}{\alpha_{h}}+\frac{1}{\lambda_{w} / d_{i}}+\frac{1}{\alpha_{c}}\right)^{-1}+\frac{1}{r+1}\left(\frac{1}{\alpha_{h} \eta_{h}}+\frac{1}{\lambda_{w} / d_{i} \cdot d_{s} / b}+\frac{1}{\alpha_{c} \eta_{c}}\right)^{-1} .
$$



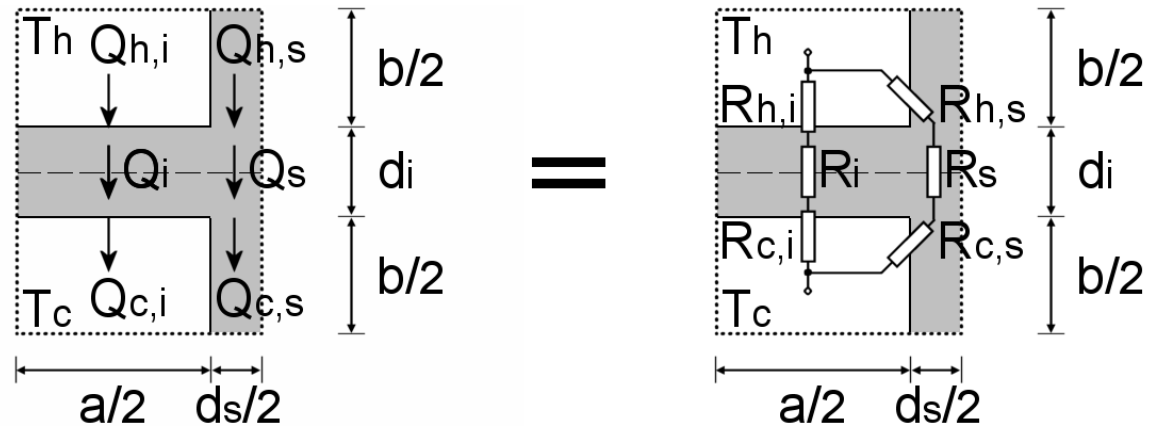

Figure 5: Model 4 - separated thermal resistances, channel side walls are treated as fins, two resistances of the intermediate and side parts of the wall between hot and cold channels.

\section{Models analysis and comparison}

Obtained values of the overall heat transfer coefficient $k$ are compared to the maximum value $k_{M A X}$ (present for wall thermal resistance equal zero):

$$
\frac{1}{k_{M A X}}=\frac{1}{\alpha_{h}}+\frac{1}{\alpha_{c}}
$$

It is assumed in this analysis that the channel size and heat transfer coefficients $\alpha_{h}, \alpha_{c}$ remain constant. The only change of the overall heat transfer coefficient $k$ is caused by the changes of wall thickness $d_{i}, d_{s}$. The common case of equal $d_{i}$ and $d_{s}$ is assumed:

$$
d=d_{i}=d_{s} .
$$

Heat transfer coefficient $\alpha$ of fluid flow can be calculated from the Nusselt number:

$$
\mathrm{Nu}=\frac{\alpha d_{h}}{\lambda_{f l}} .
$$

As an example, for fully developed laminar flow Nusselt number depends on the cross-section aspect ratio only and for $r=1$ (square minichannels) $\mathrm{Nu}=3.608[3,4]$.

There are two limiting cases of heat transfer coefficients $\alpha_{h}, \alpha_{c}$ :

- $\alpha=\alpha_{h}=\alpha_{c}$ (both the same single-phase flows), which gives $k_{M A X}=$ $\alpha / 2$, 
- $\alpha=\alpha_{h}, \alpha_{c} \rightarrow \infty$ (single-phase and two-phase flow with phase change), which gives $k_{M A X}=\alpha$.

The actual value of $k_{M A X}$ lays between these two bounds

$$
\frac{\alpha}{2} \leq k_{M A X} \leq \alpha .
$$

For each model the quotient $k / k_{M A X}$ is analysed for these two limiting cases.

A dimensionless coefficient $B$ appears in the remaining part of the analysis as

$$
B=\frac{\alpha b}{2 \lambda_{w}}=\mathrm{Nu} \frac{\lambda_{f l}}{\lambda_{w}} \frac{r+1}{4 r} .
$$

This coefficient represents properties of the wall material, fluid, channels geometry and fluid flows. For fixed cross-section aspect ratio the coefficient $B$ depends on the ratio of fluid and wall thermal conductivities only. The examples of $B$ are presented in Tab. 1.

Table 1: Examples of coefficient $B$ for square minichannels $(r=1)$, laminar flow $(\mathrm{Nu}=$ $3.608)$.

\begin{tabular}{|c|c|c|c|c|c|}
\hline No. & Wall material & Fluid & $\lambda_{w}[\mathrm{~W} / \mathrm{mK}]$ & $\lambda_{f l}[\mathrm{~W} / \mathrm{mK}]$ & $B[-]$ \\
\hline \hline 1 & copper & water & 380 & 0.60 & 0.00285 \\
\hline 2 & copper & ethanol & 380 & 0.17 & 0.000807 \\
\hline 3 & stainless steel & water & 16.3 & 0.60 & 0.0664 \\
\hline 4 & stainless steel & ethanol & 16.3 & 0.17 & 0.0188 \\
\hline 5 & ceramics & water & 1.0 & 0.60 & 1.08 \\
\hline 6 & ceramics & ethanol & 1.0 & 0.17 & 0.307 \\
\hline 7 & PE high density & water & 0.45 & 0.60 & 2.41 \\
\hline 8 & PE high density & ethanol & 0.45 & 0.17 & 0.682 \\
\hline 9 & PTFE (Teflon) & water & 0.27 & 0.60 & 4.00 \\
\hline 10 & PTFE (Teflon) & ethanol & 0.27 & 0.17 & 1.14 \\
\hline
\end{tabular}

CASE $1 \alpha=\alpha_{H}=\alpha_{C}$. For each model $k / k_{M A X}$ as a function of $B$ and relative wall thickness $d / b$ is defined:

$$
\frac{k_{1}}{k_{M A X}}=\frac{r+d / b}{r+1} \frac{1}{1+B d / b}
$$




$$
\begin{gathered}
\frac{k_{2}}{k_{M A X}}=\frac{1}{1+\frac{r+1+d / b}{r+1} B d / b}, \\
\frac{k_{3}}{k_{M A X}}=\frac{r+\eta}{r+1} \frac{1}{1+\frac{r+\eta}{r+d / b} B d / b}, \\
\frac{k_{4}}{k_{M A X}}=\frac{r}{r+1} \frac{1}{1+B d / b}+\frac{\eta}{r+1} \frac{1}{1+B \eta} .
\end{gathered}
$$

In Figs. $6-9$ calculation results of $k / k_{M A X}$ vs. relative wall thickness $d / b$ for the flow in square minichannels $(r=1, \mathrm{Nu}=3.608)$ are shown.

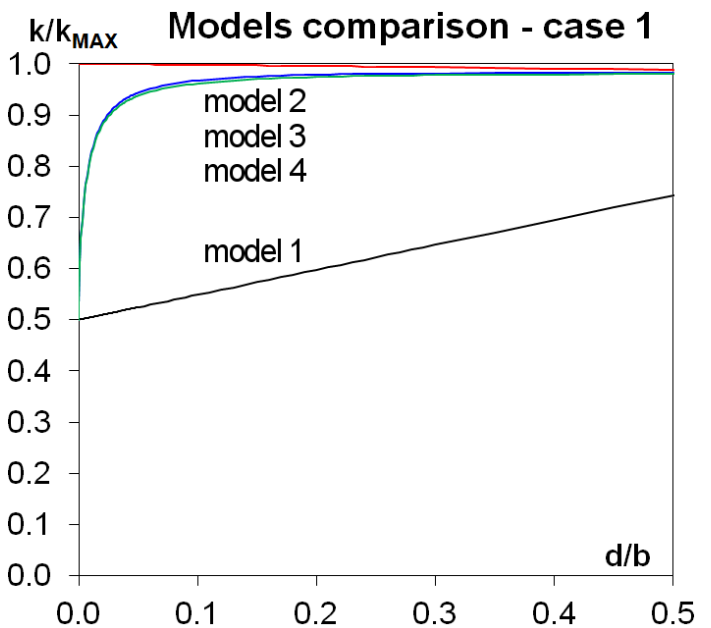

Figure 6: Models comparison for stainless steel and ethanol, $B=0.0188$.

CASE $2 \alpha=\alpha_{H}, \alpha_{C} \rightarrow \infty$

For each model $k / k_{M A X}$ as a function of $B$ and relative wall thickness $d / b$ is defined:

$$
\begin{aligned}
\frac{k_{1}}{k_{M A X}} & =\frac{r+d / b}{r+1} \frac{1}{1+2 B d / b}, \\
\frac{k_{2}}{k_{M A X}} & =\frac{1}{1+\frac{r+1+d / b}{r+1} 2 B d / b}, \\
\frac{k_{3}}{k_{M A X}} & =\frac{r+\eta}{r+1} \frac{1}{1+\frac{r+\eta}{r+d / b} 2 B d / b},
\end{aligned}
$$


$\mathrm{k} / \mathrm{k}_{\mathrm{MAX}}$ Models comparison - case 1

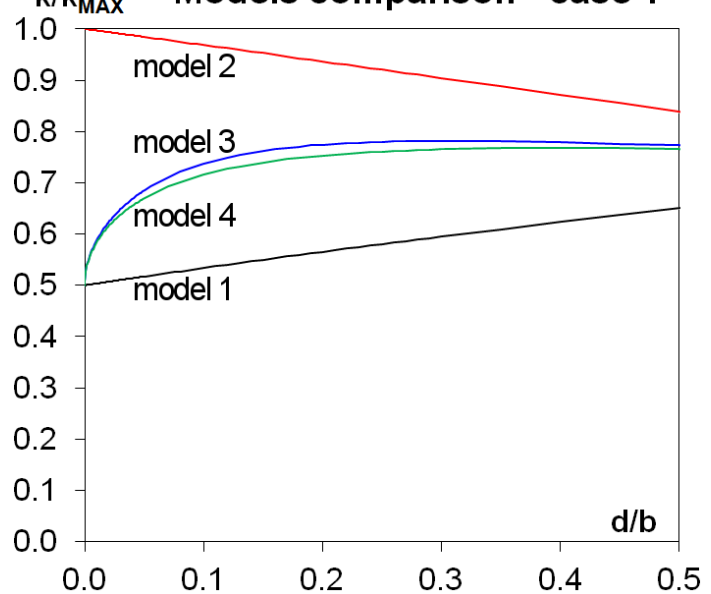

Figure 7: Models comparison for ceramics steel and ethanol, $B=0.307$.

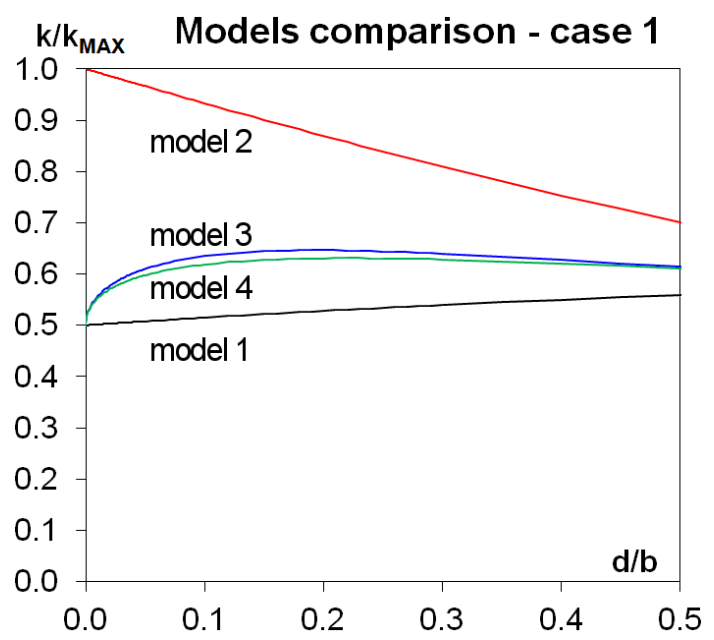

Figure 8: Models comparison for high density PE (polyethylene) and ethanol, $B=0.682$.

$$
\frac{k_{4}}{k_{M A X}}=\frac{r}{r+1} \frac{1}{1+2 B d / b}+\frac{\eta}{r+1} \frac{1}{1+2 B \eta} .
$$

It is easy to see that coefficient $B$ is replaced by $2 B$ in each formula of this case.

In Figs. $10-13$ calculation results of $k / k_{M A X}$ vs. relative wall thickness $d / b$ for the flow in square minichannels $(r=1, \mathrm{Nu}=3.608)$ are presented. 


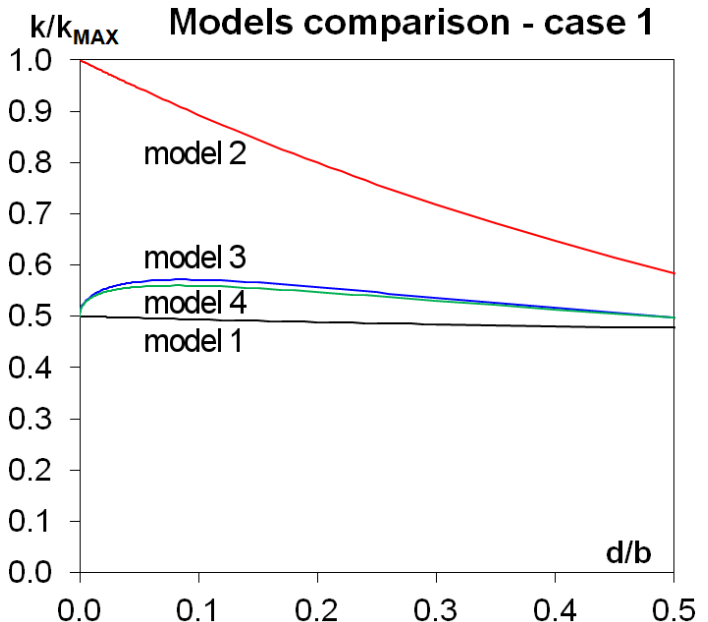

Figure 9: Models comparison for PTFE (teflon) and ethanol, $B=1.14$.

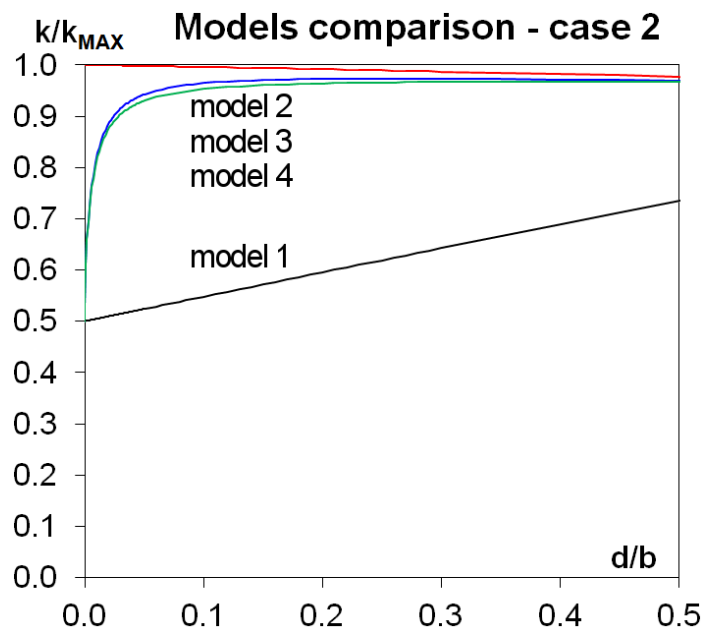

Figure 10: Models comparison for stainless steel and ethanol, $B=0.0188$. 


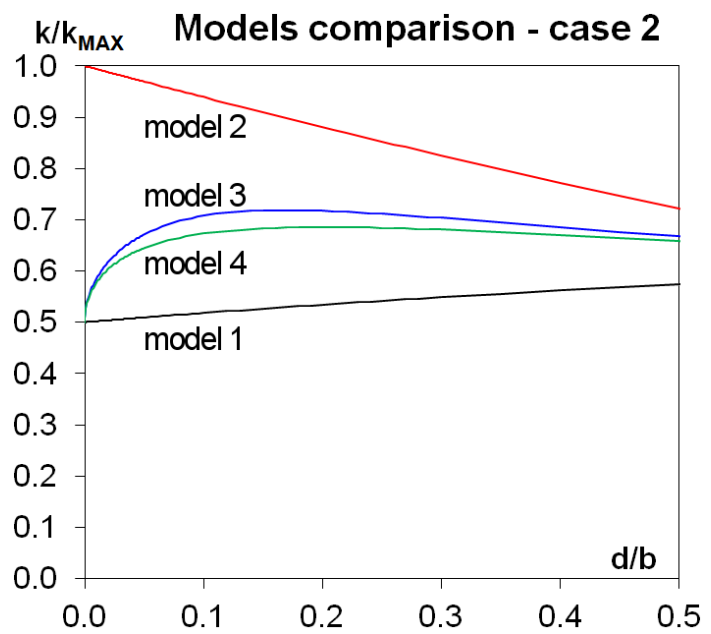

Figure 11: Models comparison for ceramics steel and ethanol, $B=0.307$.

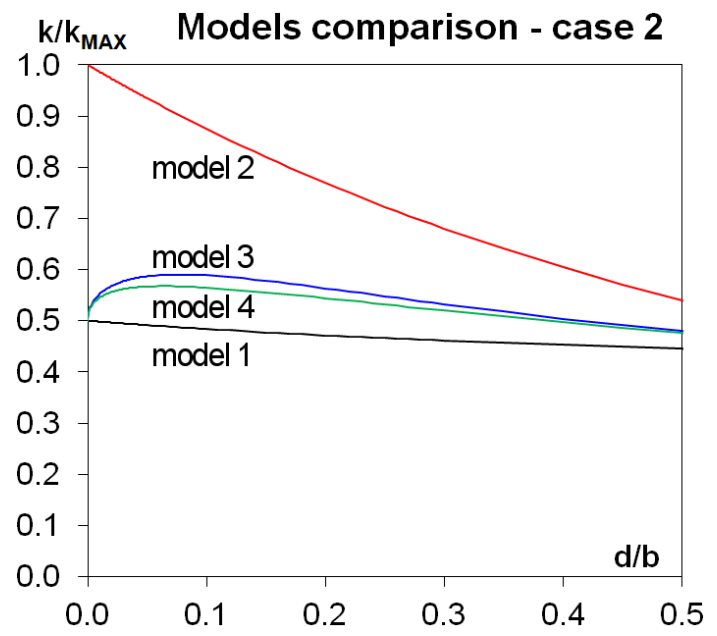

Figure 12: Models comparison for high density PE (polyethylene) and ethanol, $B=0.682$.

\section{Discussion}

Among the analysed models the most advanced are the models 3 and 4 and they give the reference results. However they are still 1-dimensional, which means that from the physical point of view, the wall material has anisotropic thermal conductivity: finite in the vertical direction and infinite 


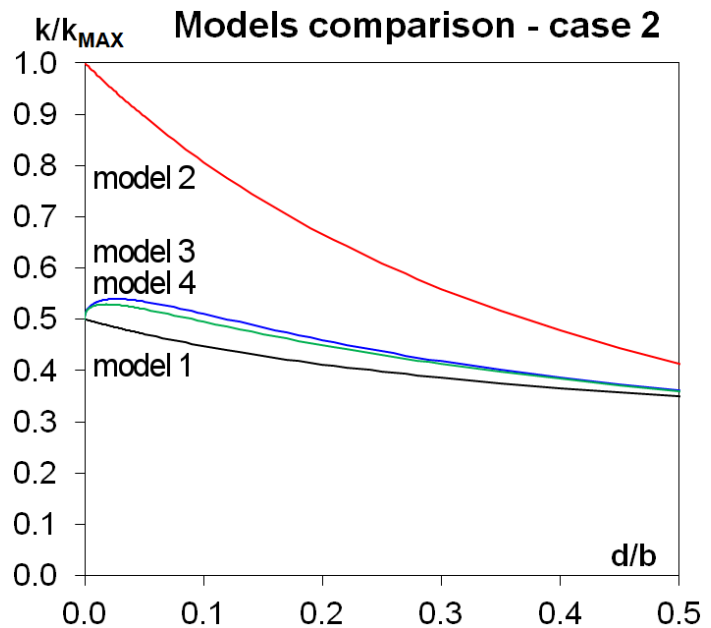

Figure 13: Models comparison for PTFE (teflon) and ethanol, $B=1.14$.

in the horizontal one. Since this artificial material has better thermal conductivity then the real one, the 1-dimensional models overestimate values of the overall heat transfer coefficient $k$.

Every realistic model used for $d / b \rightarrow 0$ should give the result

$$
\lim _{d / b \rightarrow 0} \frac{k}{k_{M A X}}=\frac{a}{a+b}=\frac{r}{r+1} .
$$

This universal result reflects the fact that infinite thin side walls do not conduct heat. For the exemplary value $r=1$ this limit is equal to $1 / 2$. Model 2 does not respect this result and does not give correct results, Figs. 6-13. Model 1 gives rough approximation of the results from models 3 and 4 for bad heat conductors like PTFE (teflon), Figs. 9 and 13, used in so called polymer heat exchangers [1]. Model 1 may be used for these materials, but the results are always slightly underestimated in comparison with models 3 and 4 . Model 4 always gives lower results of the overall heat transfer coefficient then model 3. Model 3 is more flexible than model 4 because of using single common thermal resistance $R_{i+s}$ of the wall between hot and cold minichannels instead of separate $R_{i}$ and $R_{s}$ in model 4 . Use of single $R_{i+s}$ makes possible generalization of model 3 to the case of nonequal size of hot and cold minichannels (nonequal heat transfer area $A_{h}$ and $A_{c}$ ).

Metal walls give very little thermal resistance even for relatively bad conductive stainless steel, Figs. 6 and 10. Use of typical better thermal 
conductors: brass, aluminium or copper makes wall thermal resistance negligible. For worse thermal conductors (plastics) the optimal wall thickness should be calculated.

Models 3 and 4 show the existence of optimal relative wall thickness $d / b$ maximizing $k / k_{M A X}$. The optimal value of $d / b$ is calculated by use of the condition

$$
\frac{d}{d(d / b)}\left(\frac{k}{k_{M A X}}\right)=0 .
$$

The examples of optimal values of $d / b$ for the cases 1,2 are presented in Tab. 2.

Table 2: Optimal relative thickness $d / b$ and $k / k_{M A X}$ for the cases 1 and 2 of model 3 .

\begin{tabular}{|c|l|l|l|c|c|c|c|}
\hline \multirow{2}{*}{ No. } & \multirow{2}{*}{$\begin{array}{l}\text { Wall } \\
\text { material }\end{array}$} & Fluid & B $[-]$ & \multicolumn{3}{|c|}{ Case 1} & \multicolumn{2}{|c|}{ Case 2} \\
\cline { 5 - 8 } & & & $\begin{array}{c}(d / b)_{O P T} \\
{[-]}\end{array}$ & $\begin{array}{c}\left(k / k_{M A X}\right)_{O P T} \\
{[-]}\end{array}$ & $\begin{array}{c}(d / b)_{O P T} \\
{[-]}\end{array}$ & $\begin{array}{c}\left(k / k_{M A X}\right)_{O P T} \\
{[-]}\end{array}$ \\
\hline \hline 1 & lopper & water & 0.00285 & 0.405 & 0.997 & 0.256 & 0.996 \\
\hline 2 & copper & ethanol & 0.000807 & 0.406 & 0.9992 & 0.256 & 0.9988 \\
\hline 3 & $\begin{array}{l}\text { stainless } \\
\text { steel }\end{array}$ & water & 0.0664 & 0.384 & 0.939 & 0.237 & 0.914 \\
\hline 4 & $\begin{array}{l}\text { stainless } \\
\text { steel }\end{array}$ & ethanol & 0.0188 & 0.400 & 0.982 & 0.251 & 0.973 \\
\hline 5 & ceramics & water & 1.08 & 0.0971 & 0.578 & 0.0301 & 0.543 \\
\hline 6 & ceramics & ethanol & 0.307 & 0.306 & 0.782 & 0.169 & 0.719 \\
\hline 7 & $\begin{array}{l}\text { PE high } \\
\text { density }\end{array}$ & water & 2.41 & 0.0141 & 0.520 & 0.00388 & 0.510 \\
\hline 8 & $\begin{array}{l}\text { PE high } \\
\text { density }\end{array}$ & ethanol & 0.682 & 0.192 & 0.646 & 0.0775 & 0.590 \\
\hline 9 & $\begin{array}{l}\text { PTFE } \\
\text { teflon })\end{array}$ & water & 4.00 & 0.00352 & 0.508 & 0.000923 & 0.504 \\
\hline 10 & $\begin{array}{l}\text { PTFE } \\
\text { (teflon) }\end{array}$ & ethanol & 1.14 & 0.0869 & 0.571 & 0.0265 & 0.540 \\
\hline
\end{tabular}

The optimal values of $d / b$ are fortunately small for bad thermal conductors, and the optimal side walls may still be treated as thin fins. In practice, the relative wall thickness $d / b$ should not be too small and should lay in the range $0.05-0.15[1]$.

Worse thermal conductivity of nonmetal walls may be compensated by higher number of minichannels. In the case of PTFE - teflon and ethanol 
$(B=1.14)$ the optimal $k / k_{M A X}$ is equal to 0.571 and 0.540 only, respectively in the case 1 and 2 . To compensate this, the heat transfer area should be increased by $1 / 0.571=1.75$ or $1 / 0.540=1.85$ respectively. However the side of the heat exchanger's square cross-section will be increased by smaller factors: $\sqrt{1.75}=1.32$ or $\sqrt{1.85}=1.36$ respectively. If higher number of minichannels may be accepted, the use of plastic instead of metal significantly decreases production costs.

\section{Conclusions}

- Four 1-dimensional models of thermal resistance of walls in a heat exchanger with rectangular minichannels were derived and analysed.

- Model 3 is recommended. It takes side walls into account as fins and uses single thermal resistance between layers of hot and cold minichannels. This model may be easily generalized to the case of non-equal hot and cold minichannels.

- Metal minichannel walls have negligible thermal resistance.

- The simplest model 1 may be used for plastic walls of bad thermal conductivity.

- The optimal wall thickness was derived for walls made of plastics.

- Mininichannel heat exchangers made of plastics are moderately bigger. However their production costs may be significantly lower, what is important in foreseen mass production of ORC installations used in domestic combined heat and power (CHP) technologies.

Received 23 June 2015

\section{References}

[1] Cevallos J.G., Bergles A.E., Bar-Cohen A., Rodgers P., Gupta S.K.: Polymer Heat Exchangers - History, Opportunities, and Challenges. Heat Transfer Eng. 33(2012), 13, 1075-1093.

[2] Mikielewicz D. ET AL.: Selected topics of design and investigation of heat exchangers for $O R C$ in a domestic CHP. IFFM PAS Publishers, Gdańsk 2013 (in Polish). 
[3] Rybiński W., Mikielewicz J.: Analytical solutions for laminar flow exchanging heat in rectangular channels. Arch. Thermodyn. 35(2014), 4, 29-42.

[4] Shah R.K., London A.L.: Laminar flow forced convection in ducts. Academic Press, New York 1978. 